\title{
Graphene oxide composite membranes as a potential candidate for a desalination system
}

\author{
H. Kim ${ }^{1}$, Y. H. $\mathrm{Oh}^{2}$ \& B. L. Yang ${ }^{1}$ \\ ${ }^{1}$ School of Advanced Materials and System Engineering, \\ Kumoh National Institute of Technology, Republic of Korea \\ ${ }^{2}$ Environment and Human Corporation, Republic of Korea
}

\begin{abstract}
Availability of clean water is a critical issue all over the world. In order to solve this problem, many efforts such as distillation and membrane based filtration were attempted to obtain clean water from seawater as it is an earth abundant water source. Forward osmosis research has recently been interested worldwide for desalination to supply drinking water, industrial water and agricultural water because a conventional reverse osmosis required high operating pressure which increases the running cost of desalination facilities. Currently, the graphene materials have been significantly studied because of their outstanding properties such as multifunctional surface and favourable hydrogen bonding with water molecules. In this study, we report comparative studies of commercially available forward osmosis membrane (aquaporin, sterlitech) with graphene oxides and carbon nano-tubes. The morphologies of graphene oxides and carbon nano-tubes coated aquaporin protein membranes were characterized by scanning electron microscopy. Furthermore, the salt rejection from conductivity measurement of draw solutions and the water flux of graphene oxides and carbon nano-tubes composite membranes in forward osmosis system were studied, respectively. From these results, we realized that among the characterized membrane graphene oxide coated aquaporin membrane showed higher water flux and salt rejection properties because of hydrophilic surface and functional groups such as hydroxyl $(-\mathrm{OH})$, a carboxyl $(-\mathrm{COOH})$ and an epoxide (-O-) which are hydrophilic water molecules.

Keywords: forward osmosis, graphene oxide, carbon nano-tube.
\end{abstract}




\section{Introduction}

Forward osmosis is a natural osmosis phenomenon which has got significant attraction in the development of efficient water treatment technologies as this process requires low hydraulic pressure and less electrical energy input. This is because water can spontaneously diffuse across a semi-permeable membrane by the chemical potential gradient between the feed and draw solution. However, forward osmosis membranes often show low water permeability owing to internal concentration polarization (ICP) occurring inside the porous support film of membranes and solute diffusion is another problem of these membranes. It has been realized that an ideal forward osmosis membrane should have a thin thickness, highly porous interior with a low tortuous pore structure to provide a short path from the bulk draw solution to the active layer during osmotic phenomenon, thereby alleviating the internal concentration polarization, achieving high water flux and selective layer with high dissolved solute rejection. Currently, many researchers reported the thin film composite membrane which provides the possibility to optimize support and selective layers separately. Several methods were applied to develop improve the performance of thin film composite forward osmosis membranes by controlling phase inversion process during the membrane support fabrication. Although high water permeability of thin film composite membrane with electro-spun nano-fiber substrate was reported, interfacial binding of the thin film layer substrate in such membranes is likely to be much weaker than that in the conventional membrane. In addition, greater wet-ability of the substrate is desired to improve water transport and less fouling problem. However, only a few hydrophilic polymers were used as the substrate of thin film membranes as the water might plasticize the hydrophilic support more severely than the rigid selective layer, resulting in delamination of the selective layer. The disadvantages of these polymeric membrane materials have in turn shifted the focus of research towards hydrophilic nano-composite materials [1]. In this study, we focus on the comparison of the commercial membrane with different carbonaceous materials such as carbon nano-tubes and graphene oxides. The morphologies of membranes were characterized by scanning electron microscopy. Furthermore, the salt rejection and the water flux measurements in FO system were conducted. From these results, we realized that graphene oxide coated aquaporin membrane showed higher water flux and salt rejection properties because hydrophilic surface and functional groups such as a hydroxyl $(-\mathrm{OH})$, a carboxyl (-COOH) and an epoxide (-O-) highly attracted water molecules.

\section{Materials and methods}

A 9:1 mixture of $\mathrm{H}_{2} \mathrm{SO}_{4} / \mathrm{H}_{3} \mathrm{PO}_{4}$ (Duksan) was added to a mixture of $0.375 \mathrm{~g}$ of graphite flakes (Alfa aesar) and $2.25 \mathrm{~g}$ of $\mathrm{KMnO}_{4}$ (Sigma Aldrich), producing a slight exotherm to $35-40^{\circ} \mathrm{C}$. The reaction mixture was then heated to $50^{\circ} \mathrm{C}$ and stirred for $12 \mathrm{~h}$. The reaction was cooled to room temperature at atmosphere and poured onto $50 \mathrm{ml}$ cooled deionized water with $0.375 \mathrm{ml}$ of $30 \% \mathrm{H}_{2} \mathrm{O}_{2}$ (Duksan). Regarding workup, the mixture was filtered through cellulose paper filters 
(Advantech). The filtrate was centrifuged at the $4000 \mathrm{rpm}$ for $4 \mathrm{~h}$, and the supernatant solution was decanted away. The remaining solid material was then washed successively with $200 \mathrm{~mL}$ of water, $200 \mathrm{~mL}$ of $30 \% \mathrm{HCl}$ (Duksan) and $200 \mathrm{~mL}$ of ethanol (Duksan) for each cycle. The resultant material obtained after washing process was coagulated with $200 \mathrm{~mL}$ of dimethyl ether (Alfa aesar). The graphene oxide powders were obtained by drying at $150^{\circ} \mathrm{C}$ in oven for $1 \mathrm{~h} \mathrm{[2].} \mathrm{The}$ Graphene oxide composite membranes for forward osmosis were prepared by following processes. The commercially available aquaporin protein membrane (Sterlitech) was used as polymer support and carbon nano-tube powders (Alfa aesar) were used as such without further purification. To a $20 \mathrm{mg}$ of graphene oxide, $20 \mathrm{mg}$ of carbon nano-tube were added and mixed thoroughly by dispersing in methanol (Duksan).

\section{Results}

As-prepared membranes were characterized by forward osmosis test and the setup is shown in Fig 1. This forward osmosis test setup mainly consists of a feed tank, draw tank, flow meter and forward osmosis membrane cell. The feed tank was filled out by tap water in this study. While the draw tank was filled with tap water with $3 \mathrm{M} \mathrm{NaCl}$ salts to make a chemical potential difference between feed solution and draw solution. Flow meters were installed between the solution tank and forward osmosis membrane cell to check the amount of the supplied water flux (L/min). Initially two pressure sensors were also installed but these were removed as it does not match with the circulation pumps which showed below $1 \mathrm{kgf} / \mathrm{cm}^{2}$ used in this forward test system.

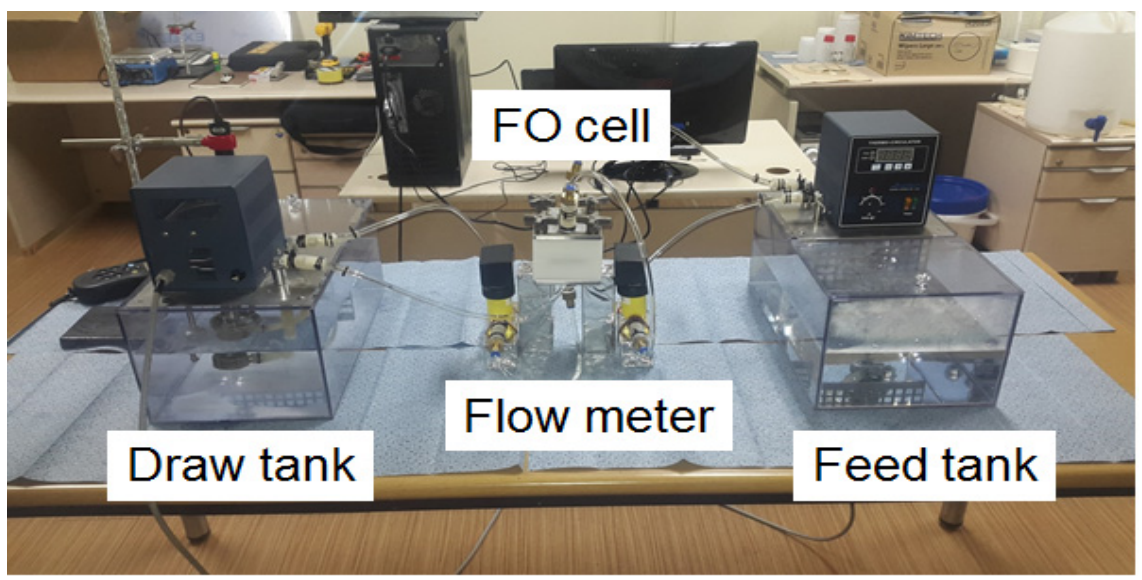

Figure 1: Experimental setup for a forward osmosis test. This is composed of a feed tank, a draw tank, flow meter and forward osmosis membrane cells (CF042, Sterlitech). 

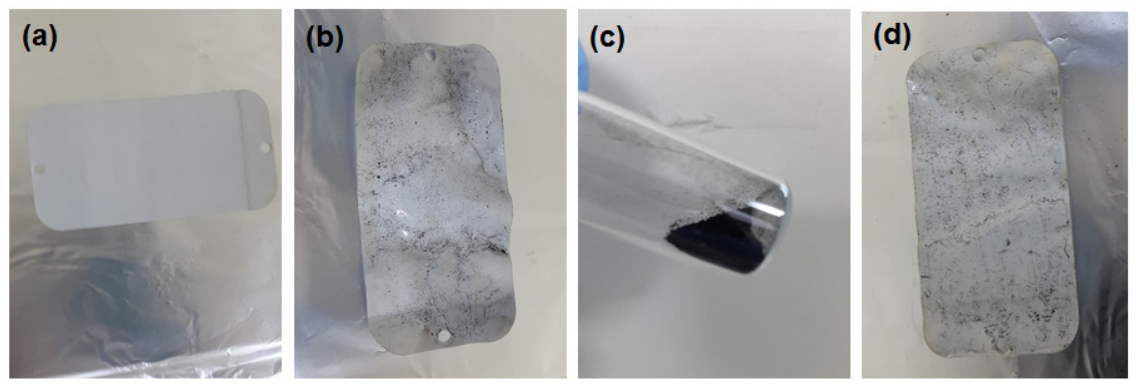

Figure 2: (a) Commercially available aquaporin membrane, (b) graphene oxide coated aquaporin membrane, (c) graphene oxide powder and (d) carbon nano-tube coated aquaporin membrane.

In this study, a commercially available forward osmosis membrane with carbon nano-materials such as graphene oxides and carbon nano-tubes was compared. The commercial membrane which is named aquaporin protein membrane is shown in Fig. 2(a). The surface of this flat membrane is smooth and the colour is white. Most of the osmosis membranes have been configured with active layer for salt rejection and support layer to be rigid membranes. It is shown in Fig. 2(b) with graphene oxide coated membrane. Graphene oxide powders were prepared by Hummer's method [3] and it is shown in Fig. 2(c). Fig. 2(d) shows the carbon nano-tubes coated membranes. As-prepared graphene oxide powders were characterized by field emission scanning electron microscopy and Raman spectroscopy and the results are shown in Fig 3(a) and (b), respectively. The microstructure image of graphene oxide showed flat and wide area with wrinkles on the surface. To confirm graphite powders were well converted into the graphene oxide, the Raman spectroscopic analysis of graphene oxide was conducted.
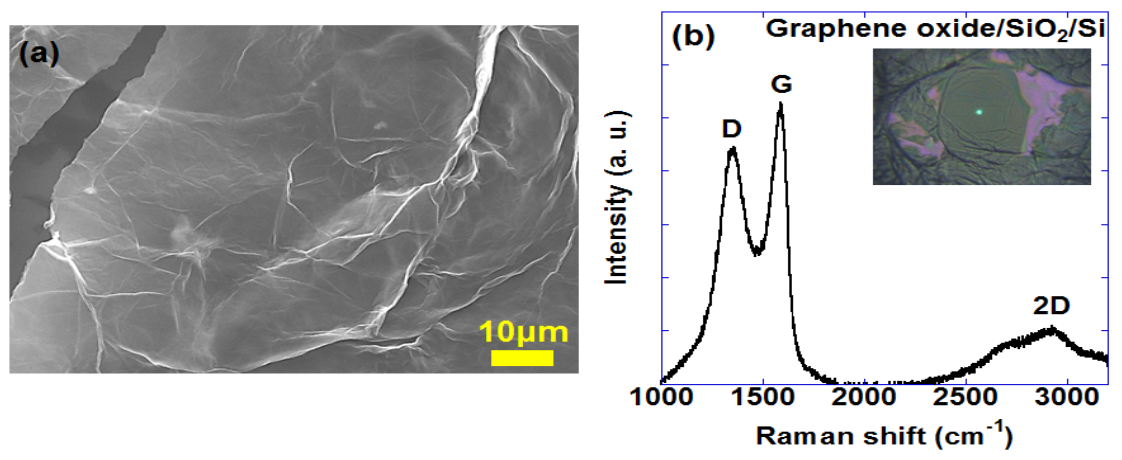

Figure 3: FE-SEM image and Raman spectroscopy of the graphene oxide on $\mathrm{SiO}_{2} / \mathrm{Si}$ substrate (a) and (b), respectively. The optical microscopic image of the graphene oxide is inserted in (b). (D peak: defects of graphene oxide, G peak: Graphite, 2D peak: a number of oxide sheets). 
The Raman shifts were scanned from the lower frequency (equal to long wave length) to the higher frequency (equal to short wave length). From the result of Raman spectroscopic analysis of graphene oxide sheets on $\mathrm{SiO}_{2} / \mathrm{Si}$ substrates, three peaks were found to be 2D peak, G peak and D peak. The first 2D peak defines a number of layers of graphene oxides. A sharp peak with a low width of the peak indicates a monolayer of graphene oxide flat sheets while broad peaks were equal to multilayer of graphene oxide flat sheets. Thus from these results, we could understand that graphene oxide was formed as flat sheets with multilayer structure. The second $\mathrm{G}$ peak defined graphite materials and its constituents. If certain carbon materials contain components such as hydrogens and oxygens or disordered carbon arrays, the intensity of the $G$ peak usually decrease and will cause a shift. Thus from these results, we could understand that uniformly ordered carbon sheets were formed with low impurities. The third D peak corresponds to a defect of the graphene oxide such as carbon vacancies, cleavages of carbon chains, folding sheets and functional groups on surface and edge of graphene oxides such as hydroxyl (-OH), an epoxide (-O-) and a carboxyl (-COOH). A high intensity of the D peak was obtained with our sample and it correspond to the above conclusion. Therefore, from these three peaks of the graphene oxide sheets it has been found that graphite powders were successfully converted graphene oxide sheets and it has got a flat sheet but a zig-zag morphology. Commercially available aquaporin protein membrane consists of active layer and the support layer which were named as asymmetric membrane configurations. Their microstructural studies together with carbon nano-tubes are shown in Fig. 4. The crosssectional image of the aquaporin membrane showed a $91 \mu \mathrm{m}$ thickness of a highly porous polyether sulfone as a support layer and a $20 \mathrm{~nm}$ of the thickness of the aquaporin as an active layer (in Fig. 4(b) and (c)). A high magnification image of the porous support layer showed their interior microstructures were consisted of thousands of branch networks. The aquaporin active surface and the polyether support layer are shown in Fig. 4(d) and 4(f), respectively. The roughness and a porosity of the active surface were found to be higher and lower than the support layer, respectively. The average pore size of the active layer was found to be $100 \mathrm{~nm}$ while the average pore size of the support layer was $0.42 \mu \mathrm{m}$. The surface image of the carbon nano-tube coated on the active layer was shown in Fig. 4(a). It showed $1 \mu \mathrm{m}$ of the average thickness and its distributions were localized on the surface. The schematic water flux in a forward osmosis was cell assembly and the results of the water flux are shown in Fig. 5(a) and 5(b), respectively. In the forward osmosis cell, Directions of the circulation of the draw solution and the feed solution containing impurities were reversely flowed each other. The diffusion direction of the net water molecule was feed side to draw side that corresponding to the diffusion direction of the low chemical potential into the high chemical potential. During the water diffusion, impurities in the feed solution were filtered on the active layer (as shown in Fig. 5(a)). The time dependence of the water flux curve was shown in Fig. 5(b). The water flux (approximately $\sim 5 \mathrm{~L} / \mathrm{h} \cdot \mathrm{m}^{2}$ ) initially increased and then it gradually decreased on reaching the top of the water flux because of the diffusion of water molecules were prohibited by rejected salts and internal concentration polarization. The water flux properties of the prepared 
(a)

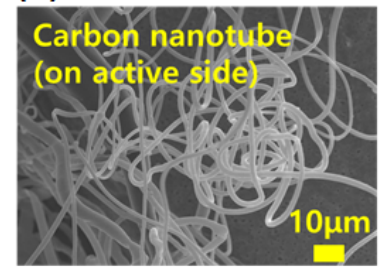

(d)

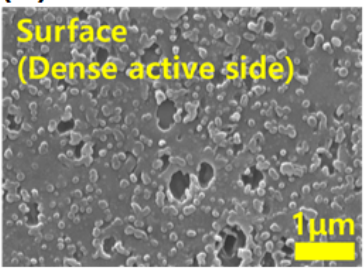

(b)

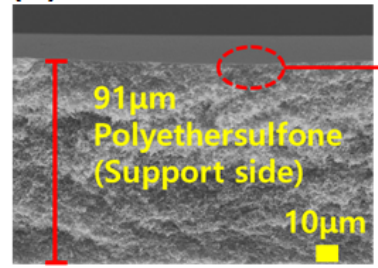

(e)

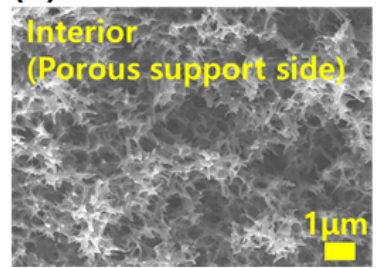

(c)

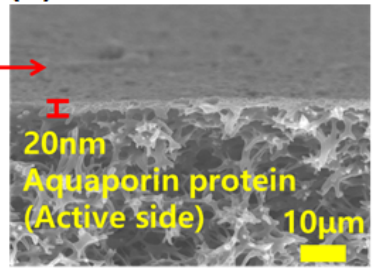

(f)

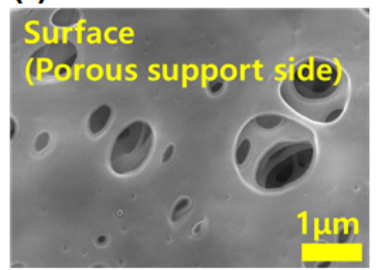

Figure 4: FE-SEM images of commercial aquaporin protein membranes and carbon nano-tube coated membrane. (a) Carbon nano-tube coated on active side of membrane, (b) and (c) cross section images of aquaporin membrane, (d) active side as an aquaporin, (e) enlarged image of the polyether sulfone as a support side and (f) porous support side of the aquaporin membrane.

(a)

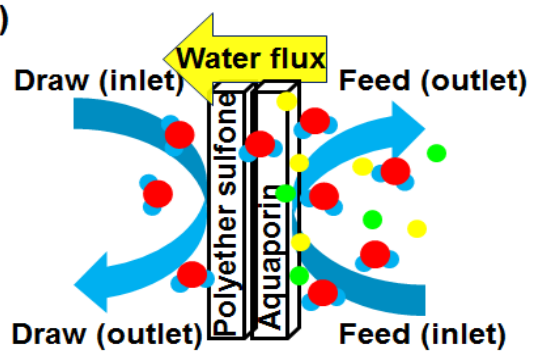

(b)

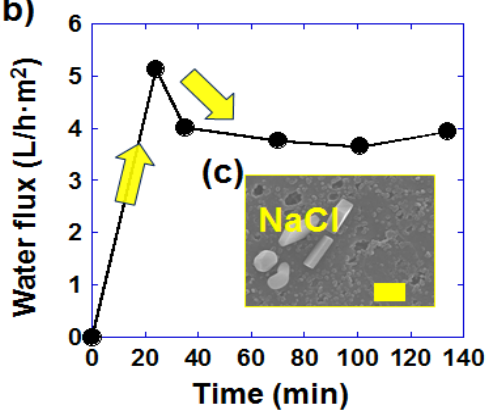

Figure 5: Schematic diagram of forward osmosis filtration of total dissolved solutes through the membrane (a), the result of water flux test (b) and filter membrane with $\mathrm{NaCl}$ crystal salts (c). (Feed solution and Draw solution were deionized water with $0.1 \mathrm{M} \mathrm{NaCl}$ and $1 \mathrm{M} \mathrm{NaCl}$ in deionized water, respectively)

membranes are shown in Fig. 6. The measured water flux of the aquaporin, graphene oxides/aquaporin and carbon nano-tubes/aquaporin was $9.8 \mathrm{~L} / \mathrm{h} \cdot \mathrm{m}^{2}$, $11.9 \mathrm{~L} / \mathrm{h} \cdot \mathrm{m}^{2}$ and $1.9 \mathrm{~L} / \mathrm{h} \cdot \mathrm{m}^{2}$ respectively. The graphene oxide coated aquaporin membrane showed a higher water flux than the others. This result indicates that functional groups such as hydroxyl (-OH), an epoxide (-O-) and a carboxyl $(-\mathrm{COOH})$ on the graphene oxide sheets make a hydrophilic surface. 


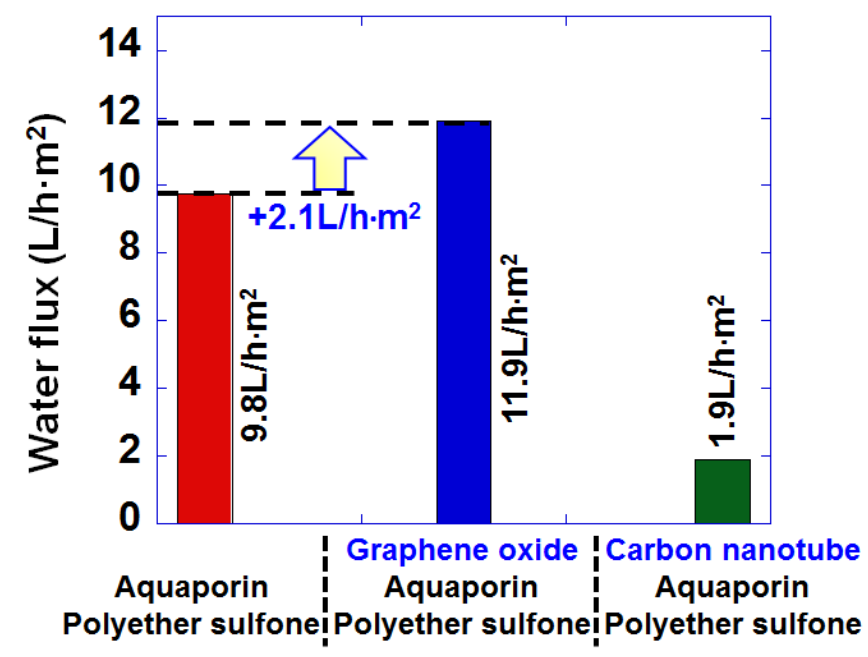

Figure 6: Water flux measurements of the bare aquaporin membrane, the graphene oxide coated aquaporin membrane and the carbon nano-tube coated aquaporin membrane. Feed solution and Draw solution were used tap water and $3 \mathrm{M} \mathrm{NaCl}$ in tap water, respectively.

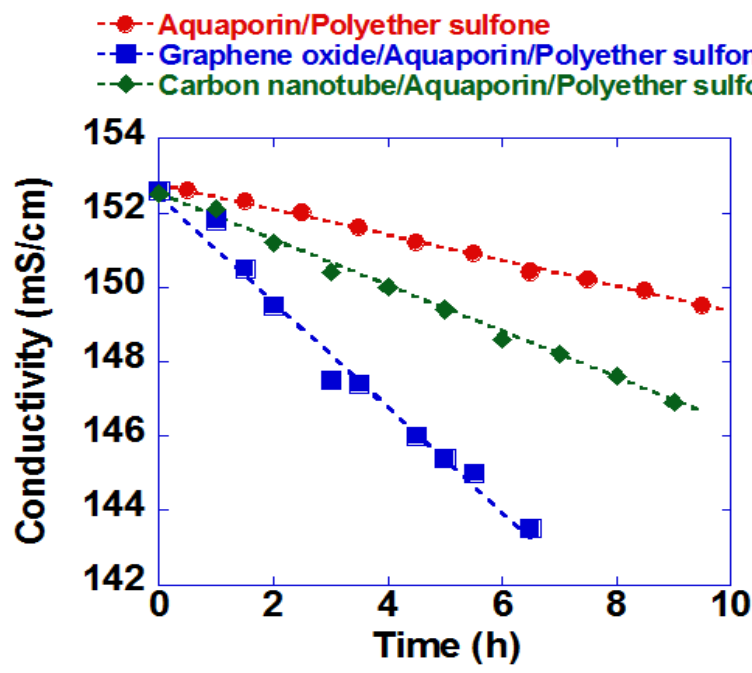

Figure 7: Conductivity measurements of the draw tank during forward osmosis operating with Aquaporin/Polyether sulfone, Graphene oxide and Carbon nano-tube coated Aquaporin membrane, respectively. 
Finally, these surfaces make it favourable for the diffusion of water molecules. Besides, increased water flux lowered the internal concentration polarization which causes adverse effects such as low water flux on membranes.

In order to characterize the salt rejection properties, an electrical conductivity of the draw solution was measured. The reducing conductivity rates of these membranes showed $0.3 \mathrm{mS} / \mathrm{cm} \cdot \mathrm{h}$ of the carbon nano-tube coated aquaporin membrane, $0.7 \mathrm{mS} / \mathrm{cm} \cdot \mathrm{h}$ of the bare aquaporin membrane and $1.5 \mathrm{mS} / \mathrm{cm} \cdot \mathrm{h}$ of the graphene oxide coated membrane.

\section{Conclusions}

In this work, forward osmosis membrane filtrations using commercially available aquaporin protein membrane were comparatively studied with that of carbon nanomaterials such as graphene oxides and carbon nano-tubes. Graphene oxide sheets were successfully synthesized from graphite powder by Hummer's method and microstructure, water flux and salt rejection properties were characterized. It was realized from these results that graphene oxide forms a hydrophilic surface on the active side which enable them to diffuse water molecules through the membrane. Therefore, to improve the filtration properties of the forward osmosis, membrane is required to have hydrophilic surface and low internal concentration polarization.

\section{Acknowledgement}

This work (Grant No. C0238693) was supported by Business for Academicindustrial Cooperative establishments funded Korea Small and Medium Business Administration in 2014.

\section{References}

[1] Y. Wang, R. Ou, H. Wang and T. Xu, "Graphene oxide modified graphitic carbon nitride as a modifier for thin film composite forward osmosis membrane," Journal of Membrane Science, 475, pp. 281-289, 2015.

[2] D. C. Marcano, D. V. Kosynkin, J. M. Berlin, A. Sinitskii, Z. Sun, A. Slesarev, L. B. Alemany, W. Lu and J. M. Tour, "Improved Synthesis of Graphene Oxide," ACS NANO, 4, pp. 4806-4814, 2010.

[3] T. Kamiya, T. J. Richardson, M.T. Flynn, A. Berliner, a. Brozel and A. Amoli, "zNANO Forward Osmosis Membrane for Wastewater Treatment Processes," 43rd International Conference on Environmental Systems, pp.112, 2013. 